\title{
Talisay (Terminalia catappa) seed husk biochar for adsorption of lead (II) ions in artificially contaminated soil
}

\author{
Joric John Canlas ${ }^{1}$, Jenika Cris $\mathrm{Go}^{1}$, Ardith Clarisse Mendoza ${ }^{1}$ and Maria Natalia Dimaano ${ }^{1,2, *}$ \\ ${ }^{1}$ Chemical Engineering Department, Faculty of Engineering, University of Santo Tomas, Philippines 1008 \\ ${ }^{2}$ Research Center for the Natural and Applied Sciences, University of Santo Tomas, España
}

\begin{abstract}
Lead, a commonly found heavy metal in contaminated soil, is highly mobilized in acidic conditions resulting to rapid soil leaching. This study investigated the effectiveness of biochar derived from Talisay (Terminalia catappa) seed husks employing the long-term immobilization of Lead in an artificially contaminated soil using simulated acid rain water meant for 100 years of rain with $5 \%$ amendment rate. Before immobilization, the heating temperatures were varied at 400,500 and $600^{\circ} \mathrm{C}$ to determine its optimal pyrolysis temperature. The biochar was pyrolyzed under three different temperatures: with 1-hour residence time. SEM images revealed that the degree of porosity of the biochar produced increased as the temperature increased. In addition, the biochar produced at $600^{\circ} \mathrm{C}$ has the highest $\%$ fixed carbon and $\%$ elemental carbon indicating stability. It then shows that Talisay seed husks are feasible materials for biochar production that can be used for the amendment of lead contaminated soil. The Freundlich and Langmuir models were used to describe the heavy metal sorption equilibrium of the biochar. Results showed that the biochar best fitted the pseudo-second-order models and Langmuir isotherm models and that the biochar displayed a favorable adsorption. Also, the immobilization experiment showed that the soil amended with biochar immobilized $\mathrm{Pb}$ decreasing its concentration by $99.47 \%$. Therefore, the produced biochar is suitable for $\mathrm{Pb}$ remediation in contaminated soils.
\end{abstract}

\section{Introduction}

Bio-charcoal (biochar) is produced when a biomass or organic material, such as wood, manure or leaves, is decomposed thermally in a closed container under a limited supply of oxygen and relatively low temperatures [1]. It is defined as a porous, finely grained substance, having a similar appearance with that of charcoal and has a relatively structured carbon matrix with high porosity and large surface area [2].

Heavy metals are persistent and difficult to remove or decompose when introduced and accumulated into soils. When left untreated, high concentrations may cause long term risks to the ecosystem and humans [3]. Hence, the treatment of heavy metals is of special concern. As an industry adapts to the biochar production technology, the agricultural benefits of biochar are to be established, thus it is essential that biochar-soil interaction be understood.

The general objective of this study is to produce a biochar from Talisay seed husk that will adsorb Lead (II) ions found Philippine soil. Pyrolyzing temperature was varied and analyzed in order to obtain the optimum temperature for the production of the biochar. Specifically, the objectives of the study are: (1) to produce Talisay seed husk and characterize it based on its features such as its porosity and morphology, as well as its degree of adsorption, (2) to determine the effects of varying temperature on the physicochemical characterization of biochar, (3) to determine the adsorption capacity and rates of the biochar and (4) to determine the effectiveness of the biochar derived from the Talisay (Terminalia catappa) seed husk through the immobilization of lead using simulated acid rainwater in an artificially contaminated soil.

\section{Materials and methods}

\subsection{Biomass and biochar production}

The dried Talisay fruit nuts were cracked using a decorticator, and the seeds were manually separated from the husks. The husks were then collected and used for this study. The collected seed husks were then milled to smaller pieces using the Thomas Wiley Laboratory Mill Model 4, with built-in $1 \mathrm{~mm}$ mesh for sieving and then subjected to another round of sieving using the USA Standard Testing Sieve ASTM E11 No. 40 of size 0.0165 $\mathrm{mm}$. Approximately 70 grams of the finally sieved biomass was contained in a closed fire assay clay crucible and subjected to pyrolysis using a muffle furnace. Pyrolytic conditions for each batch of 400, 500, and 600 ${ }^{\circ} \mathrm{C}$ with solids residence time of 1 hour were set to determine the optimum pyrolytic temperature of the biochar. The produced biochar were then cooled to room temperature, weighed, collected and labeled distinctively.

*Corresponding author: mrdimaano@gmail.com 


\subsection{Reagents}

A $1000 \mathrm{mg} / \mathrm{L}$ of $\mathrm{Pb}$ (II) stock solution was prepared by dissolving 1.59 grams of $\mathrm{Pb}\left(\mathrm{NO}_{3}\right)_{2}$ in $1 \mathrm{~L}$ of water. Acid rain was also prepared by mixing chloride forms of cations typically found in rain water. The concentration of each cation used was based on the average amount of rainfall in China [4]. Summary of the acid rainwater is given in Table 1.

Table 1. Acid Rainwater Composition [4]

\begin{tabular}{|cc|}
\hline Component & Concentration (ppm) \\
\hline $\mathbf{N H}{ }^{+}$ & 2.67 \\
\hline $\mathbf{C a}^{2+}$ & 3.37 \\
\hline $\mathbf{M g}^{\mathbf{2 +}}$ & 0.33 \\
\hline $\mathbf{K}^{+}$ & 0.79 \\
\hline $\mathbf{N a}^{+}$ & 0.36 \\
\hline
\end{tabular}

\subsection{Physical and chemical characterization of biochar}

The produced biochar from each pyrolytic condition was weighed and was compared with the initial weight of its respective raw biomass [5]. The yields expressed in the percentages of product to the raw biomass were calculated using Eq. 1.

\%Yield of Biochar $=\frac{\text { mass of biochar product }}{\text { mass of biomass }}$

Thermogravimetric analysis (TGA) was also performed to determine the proximate analysis of the biochar produced at different temperatures. The analysis was done using Perkin Elmer TGA 4000 and the method performed was adapted from the modified Donahue and Rais method. The elemental analysis of the biochar was determined using Energy Dispersive Spectroscopy (EDS) to determine the percentage amounts of $\mathrm{C}, \mathrm{N}, \mathrm{S}, \mathrm{O}$ and other trace elements in the biochar.

\subsection{Chemical characterization of soil}

The soil was subjected to elemental analysis using FEI Helios Nanolab 600i EDS, to know if there is lead present initially. The EDS was set at an accelerating voltage of $15 \mathrm{kV}$ with a beam current of $0.69 \mathrm{nA}$.

\subsection{Soil contamination}

The soil used in the study was air-dried and sieved using the USA Standard Sieve ASTM E11 No. 18. The soil contamination of lead was done by mixing 50 grams of soil and $150 \mathrm{~mL}$ of $1000 \mathrm{mg} / \mathrm{L} \mathrm{Pb}$. The suspension was subjected to rotary shaker for 24 hours. The solution was then filtered and the filtrate was subjected to Hitachi Z2300 Polarized Zeeman AAS (Atomic Absorption Spectroscopy) to determine the concentration of lead in the artificially contaminated soil. The residue was air dried for two days and then dried at $70^{\circ} \mathrm{C}$ for 4 hours and was stored for later use.

\subsection{Kinetics experiment}

To investigate the effect of time on the metal ions adsorption onto the biochar, $20 \mathrm{~mL}$ of $100 \mathrm{mg} / \mathrm{L}$ stock solution was used with 1 gram of uncontaminated soil containing $5 \%$ biochar. It was done at 1 -hour intervals for 6 hours. The suspensions were agitated using a rotary shaker at room temperature and speed of $200 \mathrm{rpm}$ constant speed. At the end of each interval, sample is withdrawn, centrifuged for 10 minutes and filtered. The filtrate was subjected to AAS to determine the specific metal content. The adsorbed heavy metal concentration was then calculated as the difference between the initial and the final metal concentration of the liquid phase.

\subsection{Adsorption isotherm}

The adsorption systems were prepared by mixing $20 \mathrm{~mL}$ of dilute solutions of $\mathrm{Pb}$ (II) at 50,100, 150 and 200 $\mathrm{mg} / \mathrm{L}$ concentrations with 1 gram of uncontaminated soil and 5\% biochar. Three trials were done for each concentration. The suspension was agitated using a rotary shaker at room temperature for 1 hour. After each trial, samples were withdrawn, centrifuged and filtered. The filtrate was subjected to AAS to determine the specific metal content.

\subsection{Lead leaching}

To determine the long-term effect of the biochar on the immobilization of lead in soil that was exposed to acid rainwater that was meant for 100 years, 2 grams of contaminated soil with $5 \%$ biochar and $20 \mathrm{~mL}$ of simulated acid rainwater, simulated for 100 years of rain, were mixed and stirred at $200 \mathrm{rpm}$ using a rotary shaker for 6 hours. The $\mathrm{pH}$ of acid rain water was not adjusted. The suspension was centrifuged and filtered. The filtrate was subjected to AAS to analyze the lead content.

\section{Results and discussion}

\subsection{Soil characteristics}

The soil sample was subjected to SEM (Scanning Electron Microscope, Hitachi TM 3000 Table Microscope) to determine its surface morphology. The SEM image of the soil sample at 5000x magnification, as shown in Figure 1, showed a uniform and flat appearance. Lead, however, can still attach itself on the exposed large surface area of the soil.

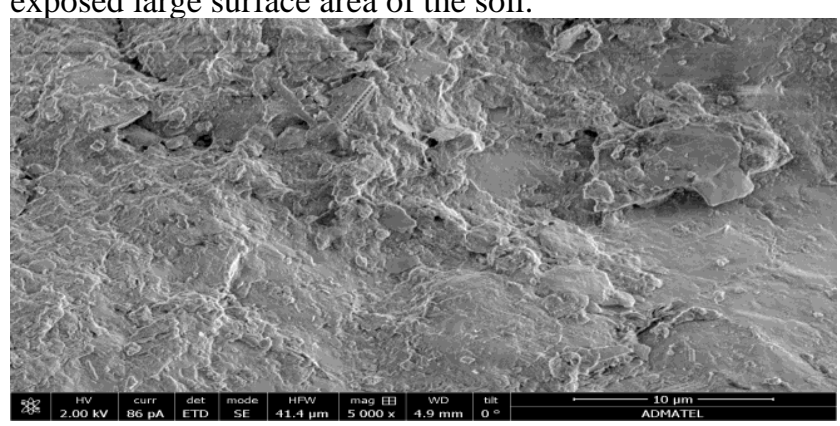

Figure 1. Soil from the UST Botanical Garden 
The elemental analysis of soil shown in Table 2 shows that the soil used has no traces of $\mathrm{Pb}$ ion.

Table 2. Elemental Analysis of Soil

\begin{tabular}{|cc|}
\hline Element & Percentage \\
\hline Oxygen & 49.63 \\
\hline Carbon & 17.23 \\
\hline Silicon & 15.87 \\
\hline Aluminum & 6.53 \\
\hline Iron & 4.87 \\
\hline Others & 5.7 \\
\hline
\end{tabular}

\subsection{Physical characterization of biochar}

The biochar produced were subjected to SEM to determine their surface morphology. The SEM images, shown in Figure 2, exhibited irregular, pattern-less appearance for all trials as well as uneven pore distribution. Among the three, the biochar produced at $600^{\circ} \mathrm{C}$ has the greatest number of openings on its surface giving lead more sites for adsorption.
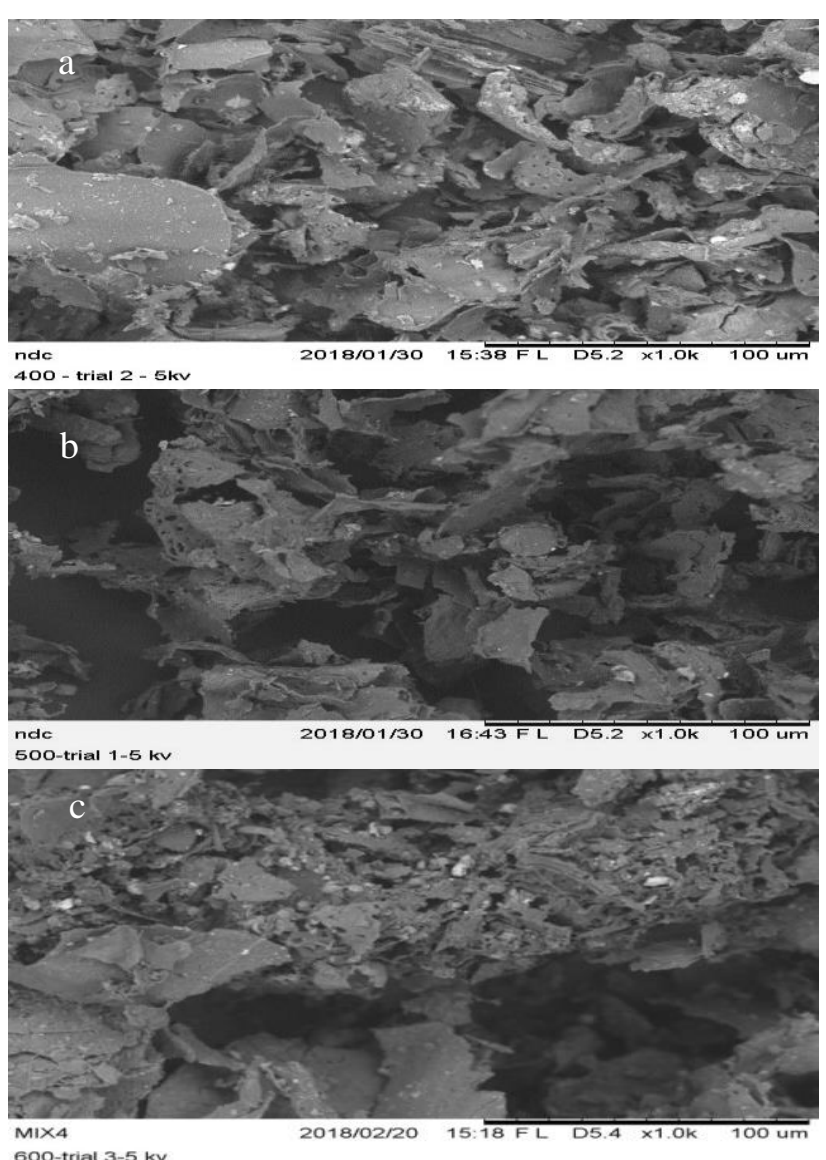

600-trial 3-5 kv

Figure 2. Comparison of SEM images of biochars produced at (a) $400^{\circ} \mathrm{C}$, (b) $500^{\circ} \mathrm{C}$ and (c) $600^{\circ} \mathrm{C}$.

\subsection{Elemental analysis of biochar}

The produced biochars were subjected to EDS to determine the elemental content. The hydrogen content, however, was not identified in the given samples due to the limitation of the equipment which can only detect elements heavier than hydrogen. Table 3 shows the Elemental Analysis of the biochar.

\subsection{Proximate analysis of biochar}

The produced biochars were subjected to TGA in order to determine their fixed carbon, volatile matter and ash contents. The proximate analyses presented in Table 4 indicates that the biochar produced at $600^{\circ} \mathrm{C}$ had the highest fixed carbon content.

Table 3. Elemental Analysis of Biochar

\begin{tabular}{|cccc|}
\hline & \multicolumn{3}{c|}{ Weight Percent } \\
\hline Element & $\mathbf{4 0 0}^{\circ} \mathbf{C}$ & $\mathbf{5 0 0}^{\circ} \mathbf{C}$ & $\mathbf{6 0 0}^{\circ} \mathbf{C}$ \\
\hline Oxygen & 44.9 & 45.87 & 12.83 \\
\hline Carbon & 20.7 & 22.1 & 78.93 \\
\hline Silicon & 14.63 & 16.57 & 0.25 \\
\hline Iron & 7.43 & 3.5 & - \\
\hline Others & 12.97 & 13.74 & 8.26 \\
\hline
\end{tabular}

Table 4. Proximate Analysis of Biochar

\begin{tabular}{|ccccc|}
\hline T $\left({ }^{\circ} \mathbf{C}\right)$ & \%M & \%FC & \%VCM & \%Ash \\
\hline $\mathbf{6 0 0}$ & 9.27 & 35.71 & 10.88 & 29.15 \\
\hline $\mathbf{5 0 0}$ & 4.16 & 14.24 & 4.15 & 71.31 \\
\hline $\mathbf{4 0 0}$ & 3.47 & 7.27 & 5.22 & 80.46 \\
\hline
\end{tabular}

\subsection{Adsorption kinetics}

A typical adsorption process includes one or more adsorbate that is physically or chemically fixed onto an adsorbent [6-7]. Figure 3 shows the effect of contact time in the adsorption of lead on the soil-biochar mixture. Majority of lead ions were adsorbed in between 1 to 2 hours, and the sorption tended toward saturation at approximately 3 hours. This phenomenon may be attributed to the instantaneous utilization of readily available adsorbing sites on the adsorbent surface [8]. The average percent removal of lead in all 3 trials for 1 hour was $99.59 \%$, indicating that a shaking time of 1 hour was suitable for the adsorption experiment.

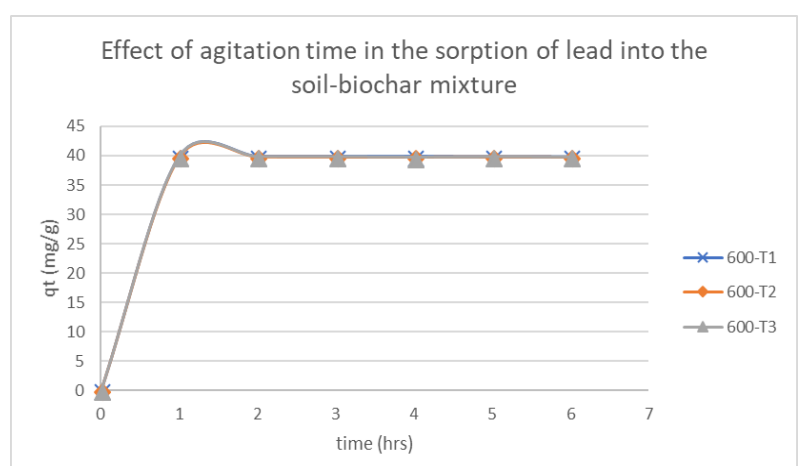

Figure 3. Effect of agitation time in the sorption of lead onto soil-biochar mixture

Lagragren's pseudo-first order (PFO) and Ho's pseudo-second order (PSO) kinetic models were applied to the experimental data to determine the adsorption 
kinetics of lead (II) ions in the soil-biochar mixture. The two models are given in their linear forms in Eq. 2 and 3, respectively:

$$
\begin{gathered}
\ln \left(Q_{e}-Q_{t}\right)=\ln Q_{e}-k_{1} t \\
\frac{\mathrm{t}}{\mathrm{Q}_{\mathrm{t}}}=\frac{1}{\mathrm{k}_{2} \mathrm{Q}_{\mathrm{e}}{ }^{2}}+\frac{1}{\mathrm{Q}_{\mathrm{e}}}
\end{gathered}
$$

where $\mathrm{Qt}=$ amount of ion adsorbed at time $\mathrm{t}(\mathrm{mg} / \mathrm{g}), \mathrm{Qe}=$ equilibrium concentration $(\mathrm{mg} / \mathrm{g}), \mathrm{k}_{1}$ and $\mathrm{k}_{2}=$ rate constants.

Figures 4 and 5 show the plots of PFO and PSO respectively. The kinetic parameters, as shown in Tables 5 and $\mathbf{6}$ were obtained by using the linearized expressions of the models. The correlation factor obtained using the PSO kinetics signifies that the chemisorption process governed the kinetics of lead (II) ions; signifying that the sorption of lead (II) ions involved precipitation and inner sphere complexation without electrostatic interaction to achieve ion exchange [9]. In addition, the equilibrium capacities obtained for the PSO were approximately equal to the experimental value further proving that it followed the PSO kinetic model.

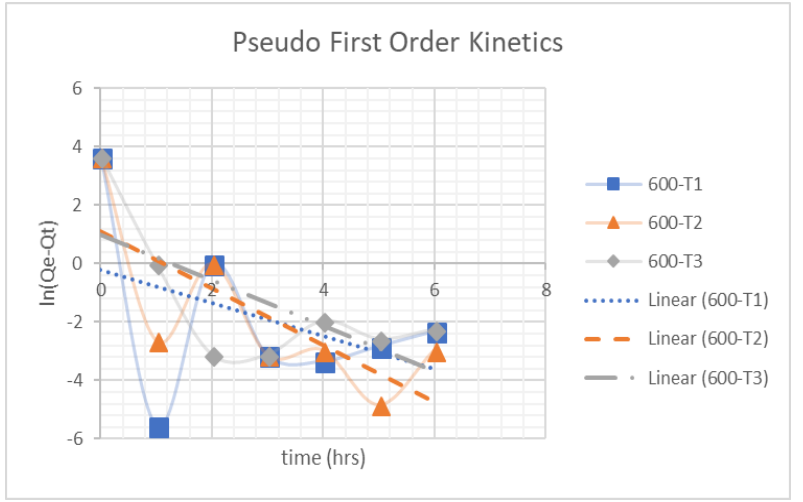

Figure 4. Pseudo- first order (PFO) graph for sorption of lead (II) ions in the soil-biochar mixture

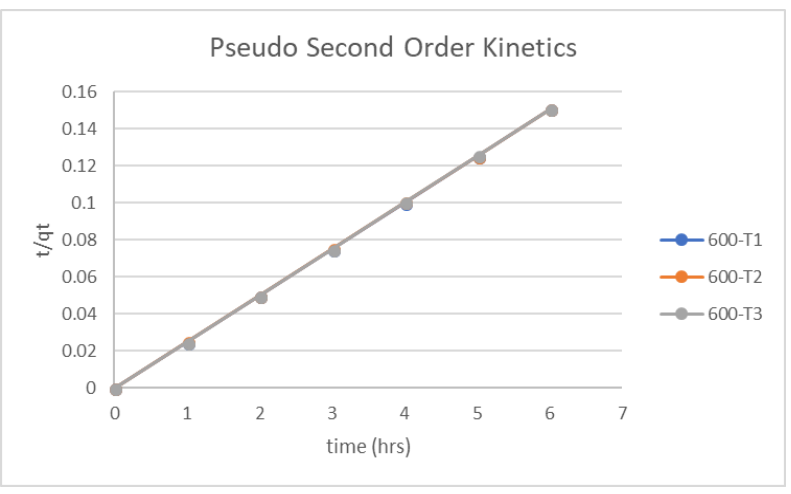

Figure 5. Pseudo-second order (PSO) graph for sorption of lead (II) ions in the soil-biochar mixture
Table 5. Kinetic Parameters of PFO

\begin{tabular}{|ccccc|}
\hline Trial & Qexperimental & $\mathbf{K 1}$ & $\mathbf{Q}_{\text {calculated }}$ & $\mathbf{R}^{\mathbf{2}}$ \\
\hline $\mathbf{1}$ & 39.88 & -0.6235 & 0.5361 & 0.1716 \\
\hline $\mathbf{2}$ & 39.832 & 0.8315 & 2.2968 & 0.5581 \\
\hline $\mathbf{3}$ & 39.872 & 1.1131 & 3.0438 & 0.4709 \\
\hline
\end{tabular}

\subsection{Adsorption isotherms}

Adsorption isotherm pertains to the relationship between the amount of adsorbed substance by an adsorbent and the equilibrium concentration of substance at constant

Table 6. Kinetic Parameters of PSO

\begin{tabular}{|ccccc|}
\hline Trial & Qexperimental & $\mathbf{K 2}$ & $\mathbf{Q}_{\text {calculated }}$ & $\mathbf{R}^{\mathbf{2}}$ \\
\hline $\mathbf{1}$ & 39.88 & 0.0251 & 39.7894 & 1 \\
\hline $\mathbf{2}$ & 39.832 & 0.0251 & 39.7934 & 1 \\
\hline $\mathbf{3}$ & 39.872 & 0.0251 & 39.7612 & 1 \\
\hline
\end{tabular}

temperature [10]. The Freundlich equation, as shown in Eq. 4, is an empirical and a widely used equation, based on the adsorption on a heterogeneous surface [11]. The equation is commonly expressed as:

$$
Q_{e}=K_{f} C_{e^{\frac{1}{n}}}
$$

and a linearized form is expressed as equation (5):

$$
\log Q_{e}=\log K_{f}+\frac{1}{n} \log C_{e}
$$

where $\mathrm{Ce}=$ equilibrium concentration $(\mathrm{mg} / \mathrm{L}), \mathrm{Qe}=$ amount of adsorbed metal ion per unit mass of adsorbent $(\mathrm{mg} / \mathrm{g}), \mathrm{K}_{\mathrm{f}}=$ Freundlich constant (relative adsorption capacity) and $1 / \mathrm{n}=$ function of strength of adsorption in the adsorption process.

The Freundlich constant signifies the relative adsorption capacity of the adsorbent, while $1 / \mathrm{n}$ measures the intensity of adsorption. The higher the $1 / \mathrm{n}$ value, the more favorable is the adsorption [13]. Figure 6 shows the plot of linearized Freundlich isotherm.

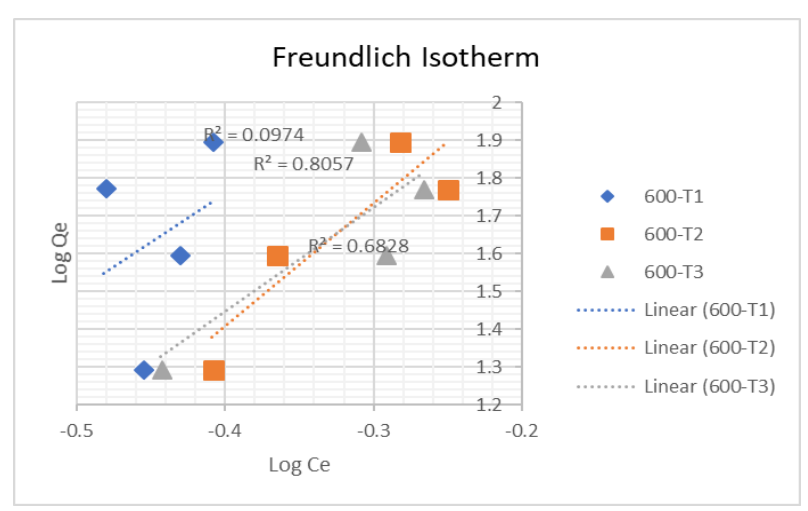

Figure 6. Plot of Freundlich Isotherm Model

Table 7 shows the Freundlich isotherm constants. Having an average value of 2.87 for $1 / \mathrm{n}$, and a value of $501.76 \mathrm{~K}_{\mathrm{f}}$, shows that a favorable adsorption occurred in the experiment. 
Table 7. Freundlich isotherm parameter

\begin{tabular}{|cccc|}
\hline Trial & $\mathbf{R}^{\mathbf{2}}$ & $\mathbf{1} / \mathbf{n}$ & $\mathbf{K}_{\mathbf{f}}$ \\
\hline $\mathbf{1}$ & 0.0974 & 2.6191 & 643.8358 \\
\hline $\mathbf{2}$ & 0.8075 & 3.2435 & 509.5769 \\
\hline $\mathbf{3}$ & 0.6828 & 2.7471 & 351.709 \\
\hline Average & $\mathbf{0 . 5 2 9 2}$ & $\mathbf{2 . 8 7}$ & $\mathbf{5 0 1 . 7 6 7 2}$ \\
\hline
\end{tabular}

The Langmuir isotherm is another popular isotherm model which also assumes that each adsorbate molecule occupies only one site, that the surface is homogenous, and that the adsorption on the surface is localized [10]. It is expressed as shown in Eq. 6 :

$$
Q_{e}=\frac{Q_{m} b C_{e}}{1+b C_{e}}
$$

and can be linearized in four ways as shown in Table 8 .

Table 8. Linearized Form of Langmuir Isotherm

\begin{tabular}{|cc|}
\hline Langmuir - 1 & $\frac{C_{e}}{Q_{e}}=\frac{C_{e}}{Q_{m}}+\frac{1}{b C_{e}}$ \\
\hline Langmuir -2 & $\frac{1}{Q_{e}}=\left(\frac{1}{b Q_{m}}\right)\left(\frac{1}{C_{e}}\right)+\frac{1}{Q_{m}}$ \\
\hline Langmuir -3 & $Q_{e}=Q_{m}-\left(\frac{1}{h}\right)\left(\frac{Q_{e}}{C_{e}}\right)$ \\
\hline Langmuir -4 & $\frac{Q_{e}}{C_{e}}=b Q_{m}-b Q_{e}$ \\
\hline
\end{tabular}

where Qe = amount of ion adsorbed $(\mathrm{mg} / \mathrm{g}), \mathrm{Ce}=$ equilibrium concentration of metal ion $(\mathrm{mg} / \mathrm{L}), \mathrm{Qm}=$ maximum amount of adsorbed metal ion per unit mass of sorbent $(\mathrm{mg} / \mathrm{g})$ and $\mathrm{b}=$ Langmuir Constant related to the rate of adsorption $(\mathrm{L} / \mathrm{mg})$.

The predicted results of $\mathrm{Qm}, \mathrm{K}_{\mathrm{L}}$ and correlation coefficient, $\mathrm{R}^{2}$, for these linearized types of Langmuir equations are shown in Table 9. It is observed in this table that Langmuir -3 and -4 have the highest average $\mathrm{R}^{2}$ value of 0.9664 , indicating that Langmuir -3 and -4 may be used to represent the lead (II) ions adsorption on the biochar. Aside from the comparison of $\mathrm{R}^{2}$ values, it was also observed that Langmuir - 1 had the highest average Qm of 12.8397. The expression of Langmuir - 1 was the most frequently used linear type to study the relation between the concentration in the solid phase and the liquid phase at equilibrium conditions [12].

The essential characteristics of the Langmuir isotherm may be expressed in terms of a dimensionless equilibrium parameter, $\mathrm{R}_{\mathrm{L}}$, and is defined by Eq. 7:

$$
R_{L}=\frac{1}{1+b C_{o}}
$$

Where $\mathrm{Co}=$ the initial metal ion concentration.

If $\mathrm{R}_{\mathrm{L}}>1$, then unfavorable adsorption occurred. $\mathrm{R}_{\mathrm{L}}=$ 1 for linear adsorption, $0<\mathrm{R}_{\mathrm{L}}<1$, indicating favorable adsorption and $\mathrm{R}_{\mathrm{L}}=0$ connotes an irreversible adsorption [12]. All 3 trials displayed favorable adsorption for either low (50 ppm) or high (200 ppm) initial concentration as shown in Table 10, indicating that the biochar produced was suitable for immobilization of lead (II) ions in soils.
Table 9. Langmuir Parameters obtained by using linear methods

\begin{tabular}{cccccc}
\hline & & Trial 1 & Trial 2 & Trial 3 & Average \\
\hline \multirow{3}{*}{ Langmuir - 1 } & $\mathrm{Qm}$ & 12.9845 & 12.1874 & 13.3473 & 12.8397 \\
\cline { 2 - 6 } & $\mathrm{b}$ & -0.0511 & -0.0577 & -0.0507 & -0.0531 \\
\cline { 2 - 6 } & $\mathrm{R}^{2}$ & 0.0507 & 0.6387 & 0.6022 & 0.4305 \\
\hline \multirow{3}{*}{ Langmuir - 2} & $\mathrm{Qm}$ & -31.171 & -15.409 & -18.553 & -21.7107 \\
\cline { 2 - 6 } & $\mathrm{b}$ & 1.5358 & -1.5316 & -1.4523 & -1.5065 \\
\cline { 2 - 6 } & $\mathrm{R}^{2}$ & 0.0612 & 0.8042 & 0.8482 & 0.5712 \\
\hline \multirow{3}{*}{ Langmuir - 3 } & $\mathrm{Qm}^{2}$ & 1.5696 & -10.665 & -5.4016 & -5.8755 \\
\cline { 2 - 6 } & $\mathrm{b}$ & 2.6772 & 1.6688 & 1.8418 & 2.0626 \\
\cline { 2 - 6 } & $\mathrm{R}^{2}$ & 0.967 & 0.9653 & 0.9669 & 0.9664 \\
\hline \multirow{3}{*}{ Langmuir -4 } & $\mathrm{Qm}^{2}$ & 3.3127 & 12.8400 & 7.2931 & 7.8153 \\
\cline { 2 - 6 } & $\mathrm{b}$ & 2.5889 & 1.1611 & 1.7808 & 1.9936 \\
\cline { 2 - 6 } & $\mathrm{R}^{2}$ & 0.967 & 0.9653 & 0.9669 & 0.9664 \\
\hline
\end{tabular}

Table 10. $R_{L}$ constant for lowest and highest initial concentration

\begin{tabular}{|ccccc|}
\hline & \multicolumn{2}{c}{ Langmuir $-\mathbf{3}$} & \multicolumn{2}{c|}{ Langmuir $-\mathbf{4}$} \\
\hline Trial & $50 \mathrm{ppm}$ & $200 \mathrm{ppm}$ & $50 \mathrm{ppm}$ & $200 \mathrm{ppm}$ \\
\hline $\mathbf{1}$ & 0.007415 & 0.001864 & 0.007666 & 0.001928 \\
\hline $\mathbf{2}$ & 0.011842 & 0.002987 & 0.012263 & 0.003094 \\
\hline $\mathbf{3}$ & 0.010742 & 0.002707 & 0.010345 & 0.002607 \\
\hline
\end{tabular}

\subsection{Lead leaching}

The AAS analysis indicated that the soil adsorbed $99.887 \%$ of $\mathrm{Pb}$ after 24 hours of mixing. The experimental results demonstrate that the $\mathrm{Pb}$ (II) concentration in the solution was reduced by $99.47 \%$. Using the student's t-test, there was a significant reduction of $\mathrm{Pb}$ indicating that the biochar was able to immobilize the lead in soil for a long period of time making it a good immobilization agent.

\section{Conclusion}

Biochar produced at $600^{\circ} \mathrm{C}$, exemplified the best adsorbing characteristic and was used for the adsorption experiments. The removal efficiency for 1 hour of the soil-biochar mixture had an average of $99.56 \%$, signifying that a run time of 1 hour was sufficient for the adsorption experiment, and a maximum utilization of readily available adsorbing sites on the adsorbent surface was evident. Both the Freundlich model and Langmuir isotherm model displayed favorable adsorption signifying that the biochar produced was suitable for immobilization of lead (II) ions in soils. While the immobilization experiment demonstrated that the biochar produced at $600^{\circ} \mathrm{C}$ was an effective immobilization agent for lead (II) ions. By using the student's t-test, a significant reduction of lead concentration was achieved by $99.47 \%$. Therefore, the biochar produced at $600^{\circ} \mathrm{C}$ is an effective adsorption and immobilization agent for lead (II) ions.

\section{References}

[1] Nartey, O. D., and Zhao, B. (2014). Biochar Preparation, Characterization, and Adsorptive Capacity and Its Effect on Bioavailability of Contaminants: An Overview. Advances in Materials Science and Engineering. 
[2] Chen, X., Chen, et al. Adsorption of copper and zinc by biochars produced from pyrolysis of hardwood and corn straw in aqueous solution. Biosource

[3] Tian-Yu Jiang, et al. Adsorption of $\mathrm{Pb}$ (II) on variable charge soils amended with rice-straw derived biochar. Chemosphere 89, 2012, 249-256.

[4] Wang, L., et al. "Impact of simulated acid rain on soil microbial community function in Masson pine seedlings." Electronic Journal of Biotechnology 17, 2014, 199-203

[5] Zhang, J., Liu, J., and Liu, R. Effects of pyrolysis temperature and heating time on biochar obtained from the pyrolysis of starw and lignosulfonate. 2015, 176.

[6] Mahtab Ahmad, et al. Effects of pyrolysis temperature on soybean stover- and peanut shellderivedbiochar properties and TCE adsorption in water.Bioresource Technology 118, 2012, 536-544

[7] Largitte, L. and R. Pasquier. "A Review of the Kinetics Adsorption Models and Their Application to the Adsorption of Lead by an Activated Carbon." ChemicalEngineering Research and Design 109, 2016, 495-504.

[8] Pehlivan, E., et al. "Lead sorption by waste biomass of hazelnut and almond shell." Journal of Hazardous Materials 167, 2009, 1203-1208.

[9] Kolodyn'ska, D., et al. "Kinetic and adsorptive characteriztion of biochar in metal ions removal." Chemical Engineering Journal 197, 2012, 295-305.

[10] Thajeel, Abbas Sabah. "Isotherm, Kinetic and Thermodynamic of Adsorption of Heavy Metal Ions onto Local Activated Carbon." Aquatic Science and Technology 1.2, 2013.

[11]Bansal, R. and Goyal, M. "Activated Carbon Adsorption" Boca Raton, 2005.

[12]Huang, Yu Ting and Ming Cheng Shih. "Effect of linearized Expressions of Langmuir Equations on the Prediction of the Adsorption of Methylene Blue on Rice Husk." International Journal of Scientific and Research Publications 6.4, 2016: 549-554.

[13] Available online at: mimoza.marmara.edu.tr/ zehra.can/ENVE401/3.\%2 0Adsorption\%20Equilibria.pdf. n.d., 2018. 